\title{
Other Caregiver
}

National Cancer Institute

\section{Source}

National Cancer Institute. Other Caregiver. NCI Thesaurus. Code C53427.

Different from the previously specified or mentioned type of person who has primary responsibility for the assistance and supervision for an individual. 